\title{
T-shaped microcantilever sensor with reduced deflection offset
}

\author{
J. A. Plaza, ${ }^{\text {a) }}$ K. Zinoviev, G. Villanueva, M. Álvarez, J. Tamayo, \\ C. Domínguez, and L. M. Lechuga \\ Centro Nacional de Microelectrónica, CSIC, Campus UAB, 08193 Cerdanyola, Barcelona, Spain
}

(Received 30 March 2006; accepted 11 July 2006; published online 31 August 2006)

\begin{abstract}
The authors have designed and fabricated arrays of microcantilevers with a geometry that shows reduced initial angular offset and angle deviation between the cantilevers of the array. This feature allows to detect the displacement of the cantilevers using the optical beam deflection technique and a single split photodetector. The structure is analytically and numerically simulated to demonstrate its feasibility. In addition, experimental measurements of the angle offset corroborate the offset and the angle deviation reduction. Finally, they illustrate the potential of these micromechanical structures as sensors by measuring a monolayer of single stranded DNA. () 2006 American Institute of Physics. [DOI: 10.1063/1.2345234]
\end{abstract}

Progress in micro- and nanotechnologies allows highly sensitive sensors to be developed with the additional advantages of miniaturization, ${ }^{1}$ robustness, reliability, low energy consumption, and mass production with the consequent reduction in costs. Silicon micromachined cantilevers have recently emerged as a promising alternative for the detection of biomolecular interactions, where the average deflection is directly related to the external interaction, usually a force or variation of the surface stress. ${ }^{2-5}$ Ultrathin microcantilevers possess low spring constants, allowing high sensitivities. ${ }^{6-8}$ The potential of these kinds of sensors relies on the combination of direct detection without the need of labeling with fluorescent molecules and the small sensor area. In the case of chemical or biological applications, microcantilever arrays enable multiple parallel experiments and thereby provide large databases. ${ }^{9-12}$ There are different methods to detect the nanomechanical response, although one of the most extended is the optical beam deflection readout, where a laser beam is focused on the free end of the cantilever. The cantilever motion is detected with subangstrom resolution by measuring the reflected beam displacement into a position-sensitive photodetector. However, the application of this technique requires cantilevers with a relatively low initial bending. Large cantilever initial curvatures induce high divergence of the reflected laser beam, significantly decreasing the sensitivity. ${ }^{9}$ This problem is even more challenging in arrays of cantilevers. In these devices, small dispersion in the curvatures of the cantilevers is also required so that a common positionsensitive photodetector can collect the laser beams reflected off all the cantilevers in the array. There are two main phenomena that can affect the initial flatness of a cantilever. First, residual stress gradient inside the structural layer induces cantilever bending. ${ }^{13}$ Single-crystalline materials, as silicon, ${ }^{14}$ can be used to solve this problem. Although this problem is already solved, there is a second origin of the initial deflection. The anchor of the cantilever to the substrate can cause stresses that induce the cantilever to rotate. ${ }^{15}$ The origin of this effect is difficult to determine and to avoid. Zhang and $\mathrm{Xu}$ proposed adjusting the curvatures of the microcantilevers after fabrication by laser bending. ${ }^{16}$ The authors claim that the initial angular offset can be adjusted by

${ }^{a)}$ FAX: +34 935801496; electronic mail: plaza@cnm.es as little as $3.5 \mu \mathrm{rad}$ for $110 \times 13 \times 0.6 \mu \mathrm{m}^{3}$ silicon cantilevers. However, the method requires measuring and adjusting every cantilever individually and only the downward precurvature can be corrected. ${ }^{16}$ Therefore, the total adjustment will be time consuming and difficult to realize for chips with arrays of cantilevers. In the present work, an easier solution to this problem is demonstrated by introducing what we call the "T-shaped cantilever." This solution allows the fabrication of extremely flat cantilevers without any additional processing. In this design, the cantilever is mechanically decoupled from the stresses at the anchor. The fabricated T-shaped cantilever consists of a stress-free single-crystalline silicon cantilever, called hereinafter a sensing cantilever, which is joined to the substrate via an intermediate doubly clamped beam, called hereinafter the supporting beam.

An analytical analysis of the T-shaped structure is performed by modeling the structure as composed of a singly clamped beam sensing cantilever, which is coupled to the substrate through a doubly clamped supporting beam. The model shows that the stresses produced at the two clamping regions of the supporting beam are relieved through parabolic deformation of the supporting beam. The deformation of the beam has a zero slope at the center where the sensing cantilever is anchored. Thus, the stresses produced at the anchoring regions do not affect the sensing cantilever. The mechanical sensitivity, related to the elastic constant, of the T-shaped structure has been analyzed and compared to the values of a rectangular cantilever with similar dimensions.

$$
\frac{k_{\mathrm{cant}}}{k_{\mathrm{T} \text {-shaped }}}=1+\frac{1}{64} \frac{w_{c}}{w_{b}}\left(\frac{L_{b}}{L_{c}}\right)^{3}+\frac{3}{2} \frac{L_{b}}{L_{c}} \frac{t^{2} w_{c}}{t^{2} w_{b}+w_{b}^{3}},
$$

where $w_{c}$ and $L_{c}$ are the width and length of the sensing cantilever (for the rectangular and the T-shaped cantilever), $w_{b}$ and $L_{b}$ the width and length of the supporting beam, and $t$ the thickness of both structures. If the dimensions of the structures are chosen such as $t$ is smaller than the other dimensions, and $w_{c} \sim w_{b}$ and $L_{c} \sim L_{b}$, it is found that the elastic constants of them are very similar. The resonant frequency of the cantilevers is calculated through the expression $\omega$ $=\sqrt{k / m}$, where $m$ is the effective mass of the structure. In order to accurately study the behavior of the structure, a finite element analysis was done using the commerciallpy available software ANSYS 9.0. A model based on material lay- 
TABLE I. Analytical and simulated results for a rectangular cantilever $\left(200 \times 20 \times 0.334 \mu \mathrm{m}^{3}\right.$, length $\times$ width $\times$ thickness $)$ and a T-shaped cantilever (beam: $100 \times 20 \times 0.334 \mu \mathrm{m}^{3}$ and cantilever: $200 \times 20 \times 0.334 \mu \mathrm{m}^{3}$ ).

\begin{tabular}{lccccc}
\hline \hline & \multicolumn{2}{c}{ Rectangular cantilever } & & \multicolumn{2}{c}{ T-shaped cantilever } \\
\cline { 2 - 3 } \cline { 6 - 6 } & Analytical & Simulated & & Analytical & Simulated \\
\hline Elastic constant, $K(\mathrm{mN} / \mathrm{m})$ & 1.967 & 1.968 & & 1.95 & 1.66 \\
Natural frequency $(\mathrm{kHz})$ & 11.486 & 11.486 & & 11.435 & 10.256 \\
\hline \hline
\end{tabular}

ers of different thermal expansion coefficients was used to simulate the stresses induced by the substrate at the anchoring points. A standard multilayer configuration for microsystems based on silicon-on-insulator (SOI) wafers was used. The first layer of single-crystalline silicon models the T-shaped cantilever. The second layer underneath was defined as silicon oxide. Finally, the bulk substrate underneath was defined as silicon. A first simulation computes the deformation of the whole structure induced by the stresses at the clamping regions of the supporting beam. As expected, these stresses deform the supporting beam, whereas the sensing cantilever remains flat. A second simulation computes the elastic constant of the T-shaped cantilever. The simulation results show that the elastic constant of the supporting beam is of about 500 times higher than that of the sensing cantilevers. Thus, only contributions of the sensing cantilever are involved and therefore it has a sensitivity similar to a rectangular cantilever. Finally, a third simulation showed that the natural frequency is also practically equal to a rectangular cantilever, due again to the major rigidity of the supporting beam with respect to the sensing cantilever. Table I includes the results obtained with both analytical and numerical simulations.

Rectangular silicon cantilevers and T-shaped cantilevers have been fabricated. The technology based on SOI wafers allows stress-free single-crystalline silicon cantilevers. Thus, the residual stresses arise mainly from the clamping regions. The dimensions of the rectangular cantilevers are $200 \times 40$ $\times 0.334 \mu \mathrm{m}^{3}$ (length $\times$ width $\times$ thickness), whereas the dimensions of the T-shaped structure are $100 \times 20$ $\times 0.334 \mu \mathrm{m}^{3}$ for the supporting beam and $200 \times 40$ $\times 0.334 \mu \mathrm{m}^{3}$ for the sensing cantilever. The sensing cantilever was designed with a paddle-shaped region at the free end for making focusing the laser beam easier. The profiles of the fabricated cantilevers were measured by confocal microscopy (model PL $\mu$ imaging profiler from Sensofar) (Fig. 1).

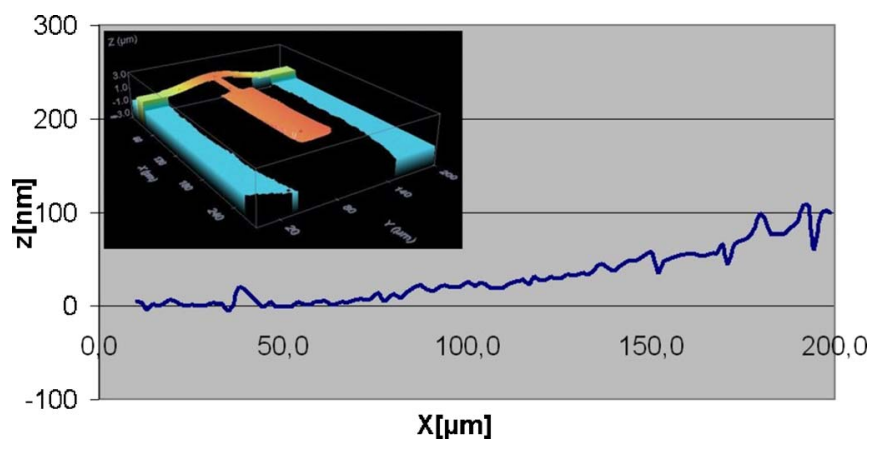

FIG. 1. Experimental results obtained with a confocal microscope of the cantilever profile and of the whole T-shaped structure due to stresses at the

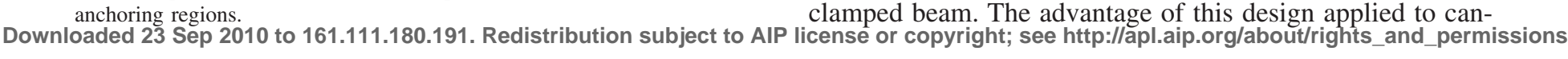

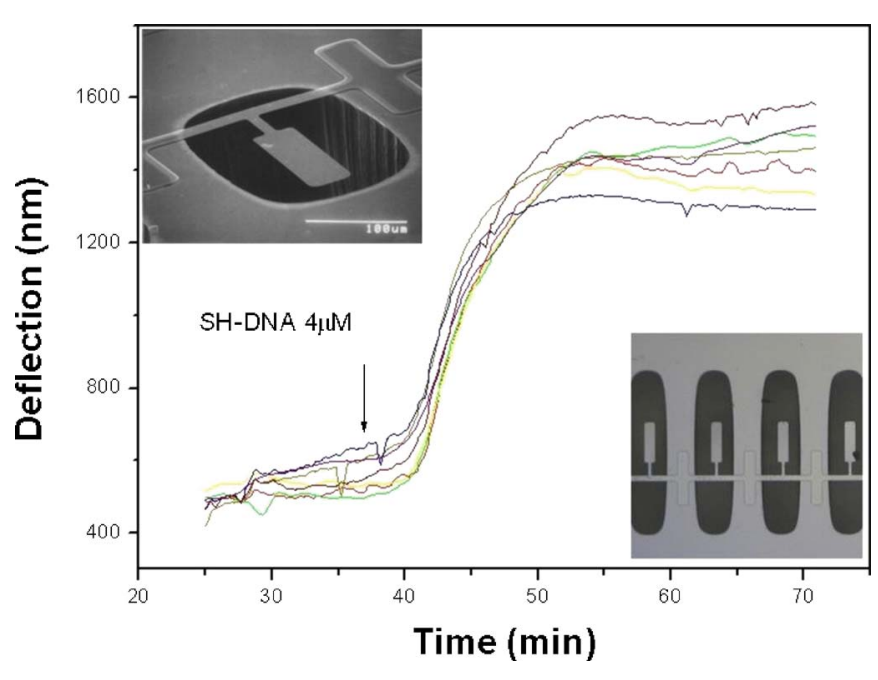

FIG. 2. Real-time deflection of seven T-shaped microcantilevers due to the immobilization of thiol modified DNA chains (SH-ssDNA). Scanning electron microscopy images of fabricated T-shaped cantilevers.

The initial deflection of an array of rectangular cantilevers in the same chip was $1045 \pm 108 \mathrm{~nm}$, whereas the deflection is reduced to $112 \pm 4 \mathrm{~nm}$ for a chip of T-shaped cantilevers. As a result, the initial deflection angle of the cantilevers was reduced by about one order of magnitude, from $(0.31 \pm 0.03)^{\circ}$ to $(0.032 \pm 0.001)^{\circ}$.

The performance of the T-shaped cantilevers was tested by measuring the cantilever response to the attachment of single stranded (ss) DNA. The adsorption of thiolated oligonucleotides provides a sufficient large signal that is little influenced by nonspecific signals due to variations of the temperature and ion concentration in solution. The simultaneous response of the cantilevers of the array was monitored by a technique based on the optical beam deflection method and the scanning of the incident laser beam across the array. This technique is described elsewhere ${ }^{15}$ and it allows the readout of the order of ten cantilevers per second with subnanometer resolution. The ssDNA immobilization was performed by using the self-assembled monolayer chemistry. ssDNA probes were 12 nucleotides in length and were synthesized with a thiol linker group, $\mathrm{SH}-\left(\mathrm{CH}_{2}\right)_{6}$ at $5^{\prime}$ end with a nucleotide sequence given by 5'-AAC GAC GGC CAG- 3' (Integrated DNA Technologies). The top side of the microcantilevers was coated with $5 \mathrm{~nm}$ of chromium and $20 \mathrm{~nm}$ of gold. The stresses produced by these coatings induce deflections on the cantilevers. However, as T-shaped cantilevers are independent of the anchor, the elastic constant of all sensing cantilevers is practically the same and hence the dispersion on the deflection of the T-shaped cantilevers is very small. Figure 2 shows the real-time deflection of seven T-shaped microcantilevers due to the immobilization of the thiol modified DNA chains (SH-ssDNA) on the gold-coated side of the cantilever. A significant bending toward the silicon side is observed, indicating that the compressive surface stress is produced on the gold surface. The deviation in the response of the seven cantilevers is related to slight variations in the surface covering, differences in the fluid diffusion and in thermal drifts.

In summary, we have designed a T-shaped structure that prevents the initial deflection of the cantilevers due to the stresses induced at the substrate clamping. The mechanical structure consists of a sensing cantilever joined to a doubly clamped beam. The advantage of this design applied to canlicense or copyright; see http://apl.aip.org/about/rights_and_permissions 
tilever arrays is that, despite possible nonuniform initial stresses at the anchor, all the cantilevers remain flat and parallel to each other. The structure can be fabricated by exactly the same technology as standard ones. This design is specially indicated for devices that involve a matrix of cantilevers for parallel optical detection, i.e., chemical or biological applications. Its application has been demonstrated by measuring a monolayer of ssDNA.

${ }^{1}$ N. V. Lavrik, M. J. Sepaniak, and P. G. Datskos, Rev. Sci. Instrum. 75, 2229 (2004).

${ }^{2}$ H. P. Lang, M. K. Baller, R. Berger, C. Gerber, J. K. Gimzewski, F. M. Battiston, P. Fornaro, J. P. Ramseyer, E. Meyer, and H. J. Guntherodt, Anal. Chim. Acta 393, 59 (1999).

${ }^{3}$ R. Raiteri, G. Nelles, H. J. Butt, W. Knoll, and P. Skladal, Sens. Actuators B 61, 213 (1999).

${ }^{4}$ J. K. Gimzewski, C. Gerber, E. Meyer, and R. R. Schlittler, Chem. Phys. Lett. 217, 589 (1994).

${ }^{5}$ E. T. Arakawa, N. V. Lavrik, S. Rajic, and P. G. Datskos, Ultramicroscopy 97, 459 (2003).

${ }^{6}$ X. X. Li, T. Ono, Y. L. Wang, and M. Esashi, Appl. Phys. Lett. 83, 3081
(2003).

${ }^{7}$ K. Y. Yasumura, T. D. Stowe, E. M. Chow, T. Pfafman, T. W. Kenny, B. C. Stipe, and D. Rugar, J. Microelectromech. Syst. 9, 117 (2000).

${ }^{8}$ M. Alvarez, J. Tamayo, J. A. Plaza, K. Zinoviev, C. Dominguez, and L. M. Lechuga, J. Appl. Phys. 99, 024910 (2006).

${ }^{9}$ M. Yue, H. Lin, D. E. Dedrick, S. Satyanarayana, A. Majumdar, A. S. Bedekar, J. W. Jenkins, and S. Sundaram, J. Microelectromech. Syst. 13, 290 (2004).

${ }^{10}$ R. McKendry, J. Y. Zhang, Y. Arntz, T. Strunz, M. Hegner, H. P. Lang, M. K. Baller, U. Certa, E. Meyer, H. J. Guntherodt, and C. Gerber, Proc. Natl. Acad. Sci. U.S.A. 99, 9783 (2002).

${ }^{11}$ J. Fritz, M. K. Baller, H. P. Lang, H. Rothuizen, P. Vettiger, E. Meyer, H. J. Guntherodt, C. Gerber, and J. K. Gimzewski, Science 288, 316 (2000).

${ }^{12}$ J. D. Adams, G. Parrott, C. Bauer, T. Sant, L. Manning, M. Jones, B. Rogers, D. McCorkle, and T. L. Ferrell, Appl. Phys. Lett. 83, 3428 (2003).

${ }^{13}$ W. Fang and J. A. Wickert, J. Micromech. Microeng. 6, 301 (1996).

${ }^{14}$ N. E. Jenkins, L. P. DeFlores, J. Allen, T. N. Ng, S. R. Garner, S. Kuehn, J. M. Dawlaty, and J. A. Marohn, J. Vac. Sci. Technol. B 22, 909 (2004).

${ }^{15}$ J. Mertens, M. Alvarez, and J. Tamayo, Appl. Phys. Lett. 87, 234102 (2005).

${ }^{16}$ X. R. Zhang and X. F. Xu, Appl. Phys. Lett. 86, 021114 (2005). 